\title{
Front Matter: Volume 7019
}

, "Front Matter: Volume 7019," Proc. SPIE 7019, Advanced Software and Control for Astronomy II, 701901 (6 August 2008); doi: 10.1117/12.805227

EDIE Event: SPIE Astronomical Telescopes + Instrumentation, 2008, Marseille, France 


\title{
PROCEEDINGS OF SPIE
}

\section{Advanced Software and Control for Astronomy II}

\author{
Alan Bridger \\ Nicole M. Radziwill \\ Editors
}

\section{6-28 June 2008}

Marseille, France

Sponsored by
SPIE
SPIE Europe

Cooperating Organizations

AAS—American Astronomical Society (USA) • ASJ_Astronomical Society of Japan (Japan)

AURA-Association of Universities for Research in Astronomy, Inc. (USA) • Ball Aerospace \& Technologies

Corporation (USA) - CNRS-Centre National de la Recherche Scientifique (France) • EAS-European

Astronomical Society (Switzerland) • ESO-European Southern Observatory (Germany) •IAU—

International Astronomical Union (France) • INSU_Institut National des Sciences de I'Univers (France)

LAM-Laboratoire d'Astrophysique de Marseille (France) - MPE-Max-Planck-Institut für

extraterrestrische Physik (Germany) - NAOJ-National Astronomical Observatory of Japan (Japan) NASA-NASA Goddard Space Flight Center (USA) • Northrop Grumman Corporation (USA) • OAMPObservatoire Astronomique de Marseille Provence (France) • OPTICON_Optical Infrared Coordination Network (United Kingdom) • RadioNet-Advanced Radio Astronomy in Europe (United Kingdom) Royal Astronomical Society (United Kingdom) • Science \& Technology Facilities Council (United Kingdom) • SFO_Société Française d'Optique (France) • Competitiveness Cluster: POPsud-Pôle Optique \& Photonique (France) $\bullet$ Optitec Sud (France)

Published by

SPIE

Volume 7019 
The papers included in this volume were part of the technical conference cited on the cover and title page. Papers were selected and subject to review by the editors and conference program committee. Some conference presentations may not be available for publication. The papers published in these proceedings reflect the work and thoughts of the authors and are published herein as submitted. The publisher is not responsible for the validity of the information or for any outcomes resulting from reliance thereon.

Please use the following format to cite material from this book:

Author(s), "Title of Paper," in Advanced Software and Control for Astronomy II, edited by Alan Bridger, Nicole M. Radziwill, Proceedings of SPIE Vol. 7019 (SPIE, Bellingham, WA, 2008) Article CID Number.

ISSN 0277-786X

ISBN 9780819472298

Published by

SPIE

P.O. Box 10, Bellingham, Washington 98227-0010 USA

Telephone +1 3606763290 (Pacific Time) · Fax +1 3606471445

SPIE.org

Copyright (C 2008, Society of Photo-Optical Instrumentation Engineers

Copying of material in this book for internal or personal use, or for the internal or personal use of specific clients, beyond the fair use provisions granted by the U.S. Copyright Law is authorized by SPIE subject to payment of copying fees. The Transactional Reporting Service base fee for this volume is $\$ 18.00$ per article (or portion thereof), which should be paid directly to the Copyright Clearance Center (CCC), 222 Rosewood Drive, Danvers, MA 01923. Payment may also be made electronically through CCC Online at copyright.com. Other copying for republication, resale, advertising or promotion, or any form of systematic or multiple reproduction of any material in this book is prohibited except with permission in writing from the publisher. The CCC fee code is $0277-786 \mathrm{X} / 08 / \$ 18.00$.

Printed in the United States of America.

Publication of record for individual papers is online in the SPIE Digital Library.

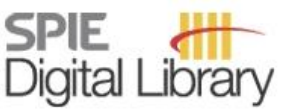

SPIEDigitallibrary.org

Paper Numbering: Proceedings of SPIE follow an e-First publication model, with papers published first online and then in print and on CD-ROM. Papers are published as they are submitted and meet publication criteria. A unique, consistent, permanent citation identifier (CID) number is assigned to each article at the time of the first publication. Utilization of CIDs allows articles to be fully citable as soon they are published online, and connects the same identifier to all online, print, and electronic versions of the publication. SPIE uses a six-digit CID article numbering system in which:

- The first four digits correspond to the SPIE volume number.

- The last two digits indicate publication order within the volume using a Base 36 numbering system employing both numerals and letters. These two-number sets start with 00, 01, 02, 03, 04, 05, $06,07,08,09,0 A, 0 B \ldots .0 Z$, followed by $10-1 Z, 20-2 Z$, etc.

The CID number appears on each page of the manuscript. The complete citation is used on the first page, and an abbreviated version on subsequent pages. Numbers in the index correspond to the last two digits of the six-digit CID number. 


\title{
Contents
}

\section{Part One}

\author{
xvii Conference Committee \\ xix Introduction \\ xxi High redshift galaxy surveys (Plenary Paper) [70 16-500] \\ M. Iye, National Astronomical Observatory of Japan (Japan)
}

\section{SESSION 1 PROJECT REPORTS: RADIO}

701902 The ALMA computing project: initial commissioning [7019-01]

B. E. Glendenning, National Radio Astronomy Observatory (United States); G. Raffi, European Southern Observatory (Germany)

701903 Software for the EVLA: current status [7019-02]

B. J. Butler, D. Harland, B. Truitt, J. Rochford, S. Witz, National Radio Astronomy Observatory (United States)

701904 Software in the CARMA heterogeneous millimeter-wave array [7019-04]

S. L. Scott, Owens Valley Radio Observatory, California Institute of Technology (United States)

\section{SESSION 2 TELESCOPE CONTROL I}

701905 A dual-consumer design for the Atacama Large Millimeter Array control subsystem [7019-05]

R. Marson, J. Kern, A. Farris, R. Hiriart, National Radio Astronomy Observatory (United States)

701906 Design and field performance of the KVN main axis control system [7019-06]

D. R. Smith, MERLAB, P.C. (United States); K. Souccar, Univ. of Massachusetts, Amherst (United States); J. Kendall, EM Interface (United States)

701907 Magdalena Ridge Observatory Interferometer: the control system of the unit telescopes [7019-07]

C. Mayer, M. Fisher, A. Greer, J. Parkhurst, P. Taylor, Observatory Sciences Ltd. (United Kingdom)

701908 Concise telescope pointing algorithm using IAU 2000 precepts [7019-08]

P. T. Wallace, STFC/Rutherford Appleton Lab. (United Kingdom)

701909 A polynomial-based trajectory generator for improved telescope control [7019-09] D. R. Smith, MERLAB, P.C. (United States); K. Souccar, Univ. of Massachusetts, Amherst (United States) 
7019 OA The software for MAGIQ: a new acquisition, guiding, and image quality monitoring system at the W. M. Keck Observatory [7019-10]

S. H. Kwok, J. Johnson, S. M. Adkins, K. McCann, W. M. Keck Observatory (United States)

7019 OC Modern computer control for Lick Observatory telescopes [7019-90]

J. Gates, W. T. S. Deich, A. Misch, R. I. Kibrick, Univ. of California Observatories, Lick

Observatory (United States)

7019 OD Instrument control tool kit for the Subaru Telescope laser guide star adaptive optics system [7019-13]

M. Dinkins, Subaru Telescope (United States)

7019 OE PCR: a PC-based wave front reconstructor for MMT-AO [7019-14]

V. Vaitheeswaran, P. Hinz, Steward Observatory, Univ. of Arizona (United States); G. Brusa,

D. Miller, Large Binocular Telescope (United States); T. Stalcup, Multiple Mirror Telescope

(United States)

\section{SESSION 4 TELESCOPE CONTROL III}

7019 OF The LBT-AdOpt arbitrator: coordinating many loosely coupled processes [7019-15]

L. Fini, F. Tosetti, L. Busoni, A. Puglisi, M. Xompero, INAF, Osservatorio Astrofisico di Arcetri (Italy)

7019 OG Applications of high-rate data logging to telescope system development and operations [7019-16]

C. J. Carter, M. J. Rippa, Gemini Observatory (United States)

$7019 \mathrm{OH} \quad$ Inspector: the GTC graphical user interface [7019-17]

R. Macías, J. M. Filgueira, GTC Project, Instituto de Astrofísica de Canarias (Spain)

7019 ol The GTC primary mirror control system [7019-18]

B. Lefort, J. Castro, GTC Project, Instituto de Astrofísica de Canarias (Spain)

7019 0J The GTC main axes servos and control system [7019-19]

M. Suárez, J. Rosich, J. Ortega, A. Pazos, GTC Project, Instituto de Astrofísica de Canarias (Spain)

7019 OK Wind Evaluation Breadboard electronics and software [7019-20]

M. Núñez, M. Reyes, T. Viera, Instituto de Astrofísica de Canarias (Spain); P. Zuluaga,

Instituto de Astrofísica de Canarias (Spain) and Altran Technologies (Spain)

\section{SESSION 5 OBSERVATORY CONTROL I}

7019 OL The STELLA robotic observatory: first two years of high-resolution spectroscopy [7019-21] M. Weber, T. Granzer, K. G. Strassmeier, M. Woche, Astrophysikalisches Institut Potsdam (Germany) 
$70190 \mathrm{M}$ Observation scheduling simulation framework: design and first results [7019-22]

S. N. Fraser, I. A. Steele, Astrophysics Research Institute, Liverpool John Moores Univ. (United Kingdom)

7019 ON The SALT observation control system [7019-23]

J. Brink, A. Charles, C. Hettlage, T.-O. Husser, A. Koeslag, E. Romero-Colmenero, South African Astronomical Observatory (South Africa)

701900 A framework for the Subaru Telescope observation control system based on the command design pattern [7019-24]

E. Jeschke, B. Bon, T. Inagaki, S. Streeper, Subaru Telescope, National Astronomical Observatory of Japan (United States)

7019 OP The ALMA/EVLA project data model: steps toward a common project description for astronomy [7019-25]

A. Bridger, UK Astronomy Technology Ctr. (United Kingdom); B. Butler, National Radio Astronomy Observatory (United States)

$70190 Q$ A common framework for the observation software of astronomical instruments at ESO [7019-108]

E. Pozna, European Southern Observatory (Germany); G. Zins, Lab. d'Astrophysique de I'Observatoire de Grenoble (France); P. Santin, INAF, Osservatorio Astronomico di Trieste (Italy); S. Beard, UK Astronomy Technology Ctr., Royal Observatory (United Kingdom)

7019 OR ALMA Observing Tool [7019-27]

A. Bridger, D. Clarke, N. P. F. Lorente, UK Astronomy Technology Ctr. (United Kingdom);

H. Yatagai, National Astronomical Observatory of Japan (Japan); M. Schilling, L. Testi,

R. H. Warmels, European Southern Observatory (Germany)

\section{SESSION 6 OBSERVATORY CONTROL II}

7019 OS Automatic validation of science programs in the Gemini Observing Tool [7019-28] A. Núñez, S. Walker, Gemini Observatory (Chile)

7019 OT KARMA: the observation preparation tool for KMOS [7019-29]

M. Wegner, B. Muschielok, Univ. Observatory Munich (Germany)

\section{SESSION 7 SOFTWARE FRAMEWORKS}

7019 OU A lightweight fault-tolerant middleware for a Subaru Telescope second generation observation control system [7019-30]

E. Jeschke, B. Bon, T. Inagaki, S. Streeper, Subaru Telescope, National Astronomical Observatory of Japan (United States)

7019 OV The Large Synoptic Survey Telescope middleware messaging system [7019-31] G. Schumacher, F. Delgado, Cerro Tololo Inter-American Observatory (Chile) 
7019 OW The ALMA common software: dispatch from the trenches [7019-32]

J. Schwarz, H. Sommer, B. Jeram, European Southern Observatory (Germany);

M. Sekoranja, CosyLAB (Slovenia); G. Chiozzi, European Southern Observatory (Germany);

A. Grimstrup, Ctr. for Radio Astronomy, Univ. of Calgary (Canada); A. Caproni, European Southern Observatory (Germany) and INAF, Osservatorio Astronomico di Trieste (Italy); C. Paredes, National Astronomical Observatory of Japan (Japan); E. Allaert, European Southern Observatory (Germany); S. Harrington, National Radio Astronomy Observatory (United States); S. Turolla, European Southern Observatory (Germany); R. Cirami, INAF, Osservatorio Astronomico di Trieste (Italy)

\section{SESSION 8 PROJECT REPORTS: OPTICAL/IR}

7019 0X Thirty Meter Telescope: observatory software requirements, architecture, and preliminary implementation strategies [7019-33]

D. R. Silva, G. Angeli, AURA/Thirty Meter Telescope (United States); C. Boyer, M. Sirota, Thirty Meter Telescope (United States); T. Trinh, JPL/Thirty Meter Telescope (United States)

7019 OY Enabling technologies and constraints for software sharing in large astronomy projects [7019-35]

G. Chiozzi, European Southern Observatory (Germany); A. Bridger, UK Astronomy Technology Ctr. (United Kingdom); K. Gillies, Space Telescope Science Institute (United States); B. Goodrich, National Solar Observatory (United States); J. Johnson, K. McCann, W. M. Keck Observatory (United States); G. Schumacher, National Optical Astronomy Observatories (Chile); S. Wampler, National Solar Observatory (United States)

\section{SESSION 9 INSTRUMENT CONTROL}

$70190 Z$ A new approach for instrument software at Gemini [7019-36]

K. Gillies, A. Nunez, Gemini Observatory (Chile); J. Dunn, Herzberg Institute of Astrophysics, National Research Council Canada (Canada)

701910 Gemini Planet Imager autonomous software [7019-37]

J. Dunn, R. Wooff, M. Smith, D. Kerley, Herzberg Institute of Astrophysics, National Research Council Canada (Canada); D. Palmer, S. Jones, Lawrence Livermore National Lab. (United States); J. Weiss, Univ. of California, Los Angeles (United States); J. Angione, Jet Propulsion Lab. (United States); J. R. Graham, Univ. of California, Berkeley (United States)

701911 The read-out and control system of the DES camera (SISPI) [7019-38] K. Honscheid, The Ohio State Univ. (United States); T. Abbott, National Optical Astronomy Observatory (United States); J. Annis, E. Buckley-Geer, Fermi National Accelerator Lab. (United States); F. Castander, Institut d'Estudis Espacials de Catalunya (Spain); J. Eiting, The Ohio State Univ. (United States); M. Gladders, Univ. of Chicago (United States); M. Haney, I. Karliner, D. Kau, Univ. of Illinois at Urbana-Champaign (United States); K. Kuehn, The Ohio State Univ. (United States); S. Kuhlmann, Argonne National Lab. (United States); T. Qian, M. Selen, J. Thaler, Univ. of Illinois at Urbana-Champaign (United States); D. Tucker, National Optical Astronomy Observatory (United States); A. Zhao, Argonne National Lab. (United States) 
701913 The NEWFIRM observing software: from design to implementation [7019-40]

P. N. Daly, N. C. Buchholz, M. J. Fitzpatrick, D. Mills, F. G. Valdes, N. Zarate, National Optical Astronomy Observatory (United States); R. Swaters, Univ. of Maryland, College Park (United States); R. G. Probst, M. Dickinson, National Optical Astronomy Observatory (United States)

701914 PyDevCom: a generic device communications application [7019-41]

S. A. McLay, UK Astronomy Technology Ctr., Royal Observatory (United Kingdom)

\section{SESSION 10 SOFTWARE ENGINEERING AND MANAGEMENT}

701916 UDP: an integral management system of embedded scripts implemented into the IMaX instrument of the Sunrise mission [7019-43]

R. Morales Muñoz, P. Mellado, Instituto de Astrofísica de Andalucía, CSIC (Spain);

J. Marco de la Rosa, Instituto de Astrofísica de Canarias (Spain)

701917 The software development process at the Chandra X-ray Center [7019-44]

J. D. Evans, I. N. Evans, G. Fabbiano, Smithsonian Astrophysical Observatory (United States)

\section{SESSION 11 DATA HANDLING AND PROCESSING I}

701918 CADOR and TAROT: a virtual observatory [7019-48]

M. Bourez-Laas, Observatoire de Haute Provence, CNRS (France); F. Vachier, Institut de Mécanique Céleste et de Calcul des Éphémérides, CNRS-OPM (France) and Observatoire de Haute Provence, CNRS (France); A. Klotz, Ctr. d'Etude Spatiale des Rayonnements, CNRS-UPS (France) and Observatoire de Haute Provence, CNRS (France); Y. Damerdji, Lab. d'Astrophysique et de Géophysique, Univ. de Liège (Belgium); M. Boër, Observatoire de Haute Provence, CNRS (France)

701919 High performance astronomical data communications in the LSST data management system [7019-46]

J. Kantor, R. Lambert, LSST Corp. (United States); C. Cox, WHREN-LILA (United States);

D. Levine, California Institute of Technology (United States); C. Smith, A. Jagatheeson,

C. Cribbs, LSST Corp. (United States)

70191 1A Data Vault: providing simple web access to NRAO data archives [7019-47]

R. DuPlain, J. Benson, E. Sessoms, National Radio Astronomy Observatory (United States)

7019 1B HERSCHEL/PACS on-board reduction flight software [7019-49]

R. Ottensamer, F. Kerschbaum, Univ. of Vienna (Austria)

SESSION 12 DATA HANDLING AND PROCESSING II

7019 1C Toward a graphical user interface for the SPIRE spectrometer pipeline [7019-50]

C. Ordenovic, C. Surace, J. Baluteau, D. Benielli, Lab. d'Astrophysique de Marseille, CNRS, Univ. de Provence (France); P. Davis, T. Fulton, Blue Sky Spectroscopy (Canada)

7019 1D Launching GUPPI: the Green Bank Ultimate Pulsar Processing Instrument [7019-53]

R. DuPlain, S. Ransom, P. Demorest, P. Brandt, J. Ford, A. L. Shelton, National Radio Astronomy Observatory (United States) 
7019 1E KISIP: a software package for speckle interferometry of adaptive optics corrected solar data [7019-54]

F. Wöger, National Solar Observatory (United States); O. von der Lühe, Kiepenhever-Institut für Sonnenphysik (Germany)

7019 IF CRBLASTER: a fast parallel-processing program for cosmic ray rejection [7019-55]

K. J. Mighell, National Optical Astronomy Observatory (United States)

\section{Part Two}

\section{INSTRUMENT CONTROL POSTER SESSION}

$70191 \mathrm{G}$ Design and implementation of a service-oriented driver architecture for LINC-NIRVANA [7019-56]

F. Kittmann, F. Briegel, L. Mohr, Max Planck Institute for Astronomy (Germany); S. Egner, Subaru Telescope, National Astronomical Observatory of Japan (United States);

W. Gaessler, J. Berwein, A. Pavlov, C. Storz, Max Planck Institute for Astronomy (Germany)

7019 1H Installation and first light of the BOOTES-IR near-IR camera [7019-57]

R. Cunniffe, A. J. Castro-Tirado, P. Kubánek, M. Jelínek, S. Vítek, J. Gorosabel,

A. de Ugarte Postigo, Instituto de Astrofísica de Andalucía, CSIC (Spain); A. Riva, F. Zerbi, Osservatorio Astronomico di Brera (Italy); A. Claret, Instituto de Astrofísica de Andalucía,

CSIC (Spain); C. Sánchez-Fernández, European Space Astronomy Ctr. (Spain)

$701911 \quad$ FPGA based control system for space instrumentation [7019-58]

A. M. Di Giorgio, P. Cerulli Irelli, F. Nuzzolo, R. Orfei, L. Spinoglio, G. S. Liu, P. Saraceno, Istituto di Fisica dello Spazio Interplanetario, INAF (Italy)

$70191 \mathrm{~K} \quad$ LBTI software architecture [7019-60]

V. Vaitheeswaran, P. Hinz, J. Kraus, Steward Observatory, Univ. of Arizona (United States);

A. Breuninger, South African Large Telescope (South Africa)

7019 1L A first-generation software product line for data acquisition systems in astronomy [7019-61] J. C. López-Ruiz, Instituto de Astrofísica de Canarias (Spain), Univ. Nacional a Distancia (Spain), and Univ. de La Laguna (Spain); R. Heradio, J. A. Cerrada Somolinos, J. R. Coz Fernandez, Univ. Nacional a Distancia (Spain); P. López Ramos, Instituto de Astrofísica de Canarias (Spain)

$70191 \mathrm{M}$ ESPRESSO control software and electronics [7019-62]

P. Di Marcantonio, P. Santin, I. Coretti, R. Cirami, M. Comari, INAF, Osservatorio

Astronomico di Trieste (Italy)

$70191 \mathrm{~N}$ The WIYN ODI instrument software architecture [7019-63]

A. K. Yeatts, WIYN Observatory (United States); D. Harbeck, WIYN Observatory (United States) and Univ. of Wisconsin, Madison (United States); J. Cavin, Univ. of Wisconsin, Madison (United States); E. McDougall, WIYN Observatory (United States)

701910 Design and realization of the control system for the three-channel birefringent filter [7019-64]

D. Zhu, Nanjing Univ. of Posts and Telecommunications (China) 
7019 1P Design and realization of the real-time spectrograph controller for LAMOST based on FPGA [7019-65]

J. Wang, National Astronomical Observatories, Nanjing Institute of Astronomical Optics \& Technology (China); L. Wu, National Astronomical Observatories, Nanjing Institute of Astronomical Optics \& Technology (China) and Graduate School of the Chinese Academy of Sciences (China); Y. Zeng, S. Dai, Z. Hu, Y. Zhu, L. Wang, Z. Wu, Y. Chen, National Astronomical Observatories, Nanjing Institute of Astronomical Optics \& Technology (China)

$70191 Q \quad$ Software structure for Vega/Chara instrument [7019-66] J.-M. Clausse, Lab. A. H. Fizeau, Observatoire de la Côte d'Azur, CNRS (France)

7019 IR The software upgrade of NICS [7019-67] E. Rossetti, INAF, Osservatorio Astronomico di Bologna (Italy); V. Guido, Univ. di Bologna (Italy) and INAF, Telescopio Nazionale Galileo (Spain); E. Oliva, INAF, Telescopio Nazionale Galileo (Spain) and INAF, Osservatorio Astrofisico di Arcetri (Italy)

7019 1S The GIANO control software system [7019-68] E. Rossetti, INAF, Osservatorio Astronomico di Bologna (Italy); E. Oliva, INAF, Osservatorio Astrofisico di Arcetri (Italy) and INAF, Telescopio Nazionale Galileo (Spain); L. Origlia, INAF, Osservatorio Astronomico di Bologna (Italy)

$70191 \mathrm{AT}$ An SOA developer framework for astronomical instrument control software [7019-70] J. Berwein, F. Briegel, W. Gaessler, Max Planck Institute for Astronomy (Germany); F. Kittmann, Univ. of Cologne (Germany); A. Pavlov, Max Planck Institute for Astronomy (Germany)

$70191 \mathrm{U}$ A component based astronomical visualization tool for instrument control [7019-71] F. Briegel, J. Berwein, Max Planck Institute for Astronomy (Germany); F. Kittmann, Max Planck Institute for Astronomy (Germany) and Univ. of Cologne (Germany); A. Pavlov, Max Planck Institute for Astronomy (Germany)

$70191 \mathrm{~V} \quad$ A very sensitive all-sky CCD camera for continuous recording of the night sky [7019-72] A. J. Castro-Tirado, M. Jelínek, Instituto de Astrofísica de Andalucía, CSIC (Spain); S. Vítek, Instituto de Astrofísica de Andalucía, CSIC (Spain) and Czech Technical Univ. (Czech Republic); P. Kubánek, Instituto de Astrofísica de Andalucía, CSIC (Spain) and Univ. de Valencia (Spain); J. M. Trigo-Rodríguez, Institut de Ciencies de l'Espai, CSIC (Spain) and Institut d'Estudis Espacials de Catalunya (Spain); A. de Ugarte Postigo, European Southern Observatory (Chile); T. J. Mateo Sanguino, Univ. de Huelva (Spain); I. Gomboš, Charles Univ. (Czech Republic)

7019 IW JMaCs: a Java monitoring and control system [7019-73]

R. Dickens, Latterfrosken Software Development Ltd. (United Kingdom)

$70191 \mathrm{X} \quad$ Workstation software framework [7019-74]

L. Andolfato, R. Karban, European Southern Observatory (Germany) 
701919 Architectural design of the control software for the SPHERE Planet Finder VLT instrument [7019-75]

A. Baruffolo, INAF, Osservatorio Astronomico di Padova (Italy); P. Bruno, INAF, Osservatorio Astrofisico di Catania (Italy); S. Cétre, Lab. d'Astrophysique de l'Observatoire de Grenoble (France); D. Fantinel, INAF, Osservatorio Astronomico di Padova (Italy); E. Fedrigo, European Southern Observatory (Germany); L. Gluck, Lab. d'Astrophysique de l'Observatoire de Grenoble (France); M. Kiekebusch, European Southern Observatory (Germany); B. Salasnich, INAF, Osservatorio Astronomico di Padova (Italy); P. Steiner, Institut für Astronomie, ETH Zürich (Switzerland); G. Zins, Lab. d'Astrophysique de l'Observatoire de Grenoble (France)

\section{TELESCOPE CONTROL POSTER SESSION}

701912 Distributed modeling and control of a segmented mirror surface [7019-76]

D. A. Kerley, Herzberg Institute of Astrophysics, National Research Council Canada (Canada); E. J. Park, Univ. of Victoria (Canada); J. Dunn, Herzberg Institute of Astrophysics, National Research Council Canada (Canada)

701920 The Large Binocular Telescope azimuth and elevation encoder system [7019-77] D. S. Ashby, Large Binocular Telescope Observatory, Univ. of Arizona (United States): T. Sargent, D. Cox, J. Rosato, Steward Observatory, Univ. of Arizona (United States);

J. G. Brynnel, Large Binocular Telescope Observatory, Univ. of Arizona (United States)

701921 The Gemini secondary mirror tip/tilt system: past, present, and future [7019-78] C. J. Carter, M. J. Rippa, Gemini Observatory (United States); R. Rojas, Gemini Observatory (Chile); M. P. Sheehan, Gemini Observatory (United States)

701922 Research and realization of message bus architecture for LAMOST control system [7019-79] L. Xu, National Astronomical Observatories, Nanjing Institute of Astronomical Optics \& Technology (China) and Graduate School of the Chinese Academy of Sciences (China)

701923 Implementation of advanced modified PCF in large telescope control system [7019-80] $X$. Shuai, National Astronomical Observatories, Nanjing Institute of Astronomical Optics \& Technology (China) and Graduate School of the Chinese Academy of Sciences (China); Z. Zhang, Y. Zhu, National Astronomical Observatories, Nanjing Institute of Astronomical Optics \& Technology (China)

701924 Research and implementation of a large telescope control system based on wireless smart sensors [7019-81]

X. Shuai, National Astronomical Observatories, Nanjing Institute of Astronomical Optics \& Technology (China) and Graduate School of the Chinese Academy of Sciences (China); Z. Zhang, National Astronomical Observatories, Nanjing Institute of Astronomical Optics \& Technology (China); C. Ren, National Astronomical Observatories, Nanjing Institute of Astronomical Optics \& Technology (China) and Graduate School of the Chinese Academy of Sciences (China); Y. Zhu, National Astronomical Observatories, Nanjing Institute of Astronomical Optics \& Technology (China) 
701925 The study on servo-control system in the large aperture telescope [7019-82]

W. Hu, National Astronomical Observatories, Nanjing Institute of Astronomical Optics \& Technology (China) and Graduate School of the Chinese Academy of Sciences (China); Z. Zhang, D. Wang, National Astronomical Observatories, Nanjing Institute of Astronomical Optics \& Technology (China)

701926 VST telescope primary mirror active optics actuators firmware implementation [7019-83] C. Molfese, INAF, VSTceN (Italy); P. Schipani, INAF, Osservatorio di Capodimonte (Italy); L. Marty, INAF, VSTceN (Italy)

701927 VST primary mirror active optics electronics [7019-84] C. Molfese, INAF, VSTceN (Italy); P. Schipani, INAF, Osservatorio Astronomico di Capodimonte (Italy); M. Capaccioli, INAF, VSTceN (Italy) and Univ. degli Studi di Napoli Federico II (Italy); G. Sedmak, Univ. degli Studi di Trieste (Italy); S. D'Orsi, INAF, VSTceN (Italy)

701928 A type of displacement actuator applied on LAMOST [7019-85]

Y. Zhang, Y. Qi, National Astronomical Observatories, Nanjing Institute of Astronomical Optics \& Technology (China)

701929 The micro-displacement worktable control system of mirror detection [7019-86] Y. Ye, National Astronomical Observatories, Nanjing Institute of Astronomical Optics \& Technology (China) and Graduate School of the Chinese Academy of Sciences (China); Z. Zhang, A. Li, National Astronomical Observatories, Nanjing Institute of Astronomical Optics \& Technology (China)

7019 2A Pre-research on arithmetic for facing control of segmented-mirror in LAMOST [7019-87] H. Miao, Nanjing Institute of Astronomical Optics \& Technology, National Astronomical Observatories (China) and Graduate School of the Chinese Academy of Sciences (China); Y. Qi, Nanjing Institute of Astronomical Optics \& Technology, National Astronomical Observatories (China)

7019 2B Servo control system for friction drive with ultra-low speed and high accuracy [7019-89] S. Yang, National Astronomical Observatories, Nanjing Institute of Astronomical Optics \& Technology (China) and Graduate School of the Chinese Academy of Sciences (China); Z. Zhang, National Astronomical Observatories, Nanjing Institute of Astronomical Optics \& Technology (China)

7019 2C Gemini all-sky camera for laser guide star operation [7019-91] M. Bec, F. J. Rigaut, G. Trancho, M. Boccas, F. Collao, F. Daruich, C. d'Orgeville, M. Lazo, D. Maltes, G. Perez, V. Vergara, T. Vucina, Gemini Observatory (Chile); M. P. Sheehan, Gemini Observatory (United States)

7019 2D Improved guiding accuracy through slit viewer of Subaru Telescope [7019-92] A. Iseki, D. Tomono, A. Tajitsu, Subaru Telescope, National Astronomical Observatory of Japan (Japan); N. Itoh, S. Miki, Mitsubishi Electric Corp. (Japan)

$70192 \mathrm{~F} \quad$ Oeil 1.0 visual control system for the pointing and tracking of ground-based telescopes [7019-95]

J. Rodríguez Ferreira, Á. Gélvez Espinel, A. Plata Gómez, Univ. Industrial de Santander (Colombia); J. Mejía Cabeza, Instituto Nacional de Pesquisas Espaciais (Brazil) 
$701921 \quad$ An amateur telescope control system: toward a generic telescope control model [7019-99] R. J. Tobar, H. H. von Brand, M. A. Araya, J. S. López, Univ. Técnica Federico Santa María (Chile)

7019 2J A nonlinear disturbance-decoupled elevation axis controller for the Multiple Mirror Telescope [7019-100]

D. Clark, T. Trebisky, Multiple Mirror Telescope Observatory, Univ. of Arizona (United States);

K. Powell, Steward Observatory Ctr. for Astronomical Adaptive Optics, Univ. of Arizona (United States)

\section{OBSERVATORY CONTROL POSTER SESSION}

7019 2K OAdM robotic observatory: solutions for an unattended small-class observatory [7019-101]

J. Colomé, I. Ribas, Institut d'Estudis Espacials de Catalunya (Spain) and Institut de Ciències de I'Espai, CSIC-IEEC (Spain); D. Fernández, Institut d'Estudis Espacials de Catalunya (Spain) and Institut de Ciències del Cosmos, UB-IEEC (Spain); X. Francisco, Institut d'Estudis Espacials de Catalunya (Spain); J. Isern, Institut d'Estudis Espacials de Catalunya (Spain) and Institut de Ciències de I'Espai, CSIC-IEEC (Spain); X. Palau, Fundació Joan Oró (Spain); J. Torra, Institut d'Estudis Espacials de Catalunya (Spain) and Institut de Ciències del Cosmos, UB-IEEC (Spain)

7019 2L Systems and control software for the Atacama Cosmology Telescope [7019-102] E. R. Switzer, Princeton Univ. (United States); C. Allen, NASA Goddard Space Flight Ctr. (United States); M. Amiri, The Univ. of British Columbia (Canada); J. W. Appel, Princeton Univ. (United States); E. S. Battistelli, Univ. of Rome La Sapienza (Italy) and The Univ. of British Columbia (Canada); B. Burger, The Univ. of British Columbia (Canada); J. A. Chervenak, NASA Goddard Space Flight Ctr. (United States); A. J. Dahlen, S. Das, Princeton Univ. (United States); M. J. Devlin, S. R. Dicker, Univ. of Pennsylvania (United States); W. B. Doriese, National Institute of Standards and Technology (United States); R. Dünner, Pontificia Univ. Católica de Chile (Chile) and Princeton Univ. (United States); T. Essinger-Hileman, Princeton Univ. (United States); X. Gao, UK Astronomy Technology Ctr., Royal Observatory (United Kingdom); M. Halpern, M. Hasselfield, The Univ. of British Columbia (Canada); G. C. Hilton, National Institute of Standards and Technology (United States); A. D. Hincks, Princeton Univ. (United States); K. D. Irwin, National Institute of Standards and Technology (United States); S. Knotek, The Univ. of British Columbia (Canada); R. P. Fisher, J. W. Fowler, N. Jarosik, Princeton Univ. (United States); M. Kaul, J. Klein, Univ. of Pennsylvania (United States); J. M. Lau, Stanford Univ. (United States); M. Limon, Columbia Astrophysics Lab. (United States); R. H. Lupton, T. A. Marriage, Princeton Univ. (United States); K. L. Martocci, City Univ. of New York (United States); S. H. Moseley, NASA Goddard Space Flight Ctr. (United States); C. B. Netterfield, Univ. of Toronto (Canada); M. D. Niemack, Princeton Univ. (United States); M. R. Nolta, Canadian Institute for Theoretical Astrophysics, Univ. of Toronto (Canada); L. Page, L. P. Parker, B. A. Reid, Princeton Univ. (United States); C. D. Reintsema, National Institute of Standards and Technology (United States); A. J. Sederberg, Princeton Univ. (United States); J. L. Sievers, Canadian Institute for Theoretical Astrophysics, Univ. of Toronto (Canada); D. N. Spergel, S. T. Staggs, O. R. Stryzak, Princeton Univ. (United States);

D. S. Swetz, R. J. Thornton, Univ. of Pennsylvania (United States); E. J. Wollack, NASA Goddard Space Flight Ctr. (United States); Y. Zhao, Princeton Univ. (United States)

$70192 \mathrm{M} \quad$ Achieving design reuse: a case study [7019-103]

P. J. Young, J. J. Nielsen, W. H. Roberts, G. M. Wilson, Research School of Astronomy and Astrophysics, Australian National Univ., Mount Stromlo Observatory (Australia) 
7019 2N Gathering headers in a distributed environment [7019-104]

B. D. Goodrich, S. B. Wampler, J. R. Hubbard, National Solar Observatory (United States)

701920 Observation process of LAMOST using observatory control system: testing for the command model and interface agent [7019-105]

S. W. Sun, A-L. Luo, National Astronomical Observatories (China)

$70192 \mathrm{P} \quad$ Software design for the control system of small LAMOST [7019-106]

S. Yang, National Astronomical Observatories, Nanjing Institute of Astronomical Optics \& Technology (China) and Graduate School of the Chinese Academy of Sciences (China)

$70192 \mathrm{Q}$ Optimizing real-time web-based user interfaces for observatories [7019-107]

J. D. Gibson, T. E. Pickering, D. Porter, S. Schaller, MMT Observatory, Univ. of Arizona (United States)

7019 2R Implementation of the software systems for the SkyMapper automated survey telescope [7019-109]

A. Vaccarella, T. Preston, A. Czezowski, S. C. Keller, B. P. Schmidt, P. J. Young, Research

School of Astronomy and Astrophysics, Australian National Univ., Mount Stromlo

Observatory (Australia)

701925 The RTS2 protocol [7019-110]

P. Kubánek, GACE, Univ. de Valencia (Spain) and Instituto de Astrofísica de Andalucía,

CSIC (Spain); M. Jelínek, Instituto de Astrofísica de Andalucía, CSIC (Spain); J. French, Univ. College Dublin (Ireland); M. Prouza, Fyzikální ústav Akademie Věd (Institute of

Physics) (Czech Republic); S. Vítek, A. J. Castro-Tirado, Instituto de Astrofísica de Andalucía, CSIC (Spain); V. Reglero, GACE, Univ. de Valencia (Spain)

7019 2T The control and data concept for the robotic solar telescope ChroTel [7019-111] C. Halbgewachs, Ch. Bethge, P. Caligari, Kiepenhever-Institut für Sonnenphysik (Germany); D. Elmore, High Altitude Observatory, National Ctr. for Atmospheric Research (United States); T. J. Kentischer, H. Peter, M. Sigwarth, W. Schmidt, Kiepenhever-Institut für Sonnenphysik (Germany)

$70192 \mathrm{U} \quad$ PLATO control and robotics [7019-113]

D. M. Luong-Van, M. C. B. Ashley, J. R. Everett, J. S. Lawrence, J. W. V. Storey, Univ. of New South Wales (Australia)

7019 2V Controlling the Hamburg Robotic Telescope: a description of the software [7019-114] J. N. González-Pérez, A. Hempelmann, M. Mittag, H.-J. Hagen, Hamburger Sternwarte (Germany)

\section{SOFTWARE ENGINEERING POSTER SESSION}

7019 2W Virtualization as an alternative for astronomical software integration [7019-115] L. A. Martínez, A. Bernal, F. Garfias, Instituto de Astronomía, Univ. Nacional Autónoma de México (Mexico) 
7019 2X Software regression testing: practical experience at the ALMA test facility [7019-116]

B. Lopez, R. Araya, N. Barriga, P. Burgos, Atacama Large Millimeter Array (Chile);

S. Harrington, T. Juerges, J. Kern, National Radio Astronomy Observatory (United States);

J. Sepulveda, R. Soto, N. Troncoso, M. Zambrano, Atacama Large Millimeter Array (Chile)

$70192 Y \quad$ A case of reliable remote functionality [7019-117]

G. Lin, National Astronomical Observatories (China)

\section{DATA HANDLING POSTER SESSION}

$70192 Z \quad$ Storage options for petabytes of data [7019-118]

L. Paton, Smithsonian Astrophysical Observatory (United States); J. Cirtain, NASA Marshall Space Flight Ctr. (United States); P. Grant, Smithsonian Astrophysical Observatory (United States)

701930 SPHERE baseline software for reducing calibration data [7019-120]

M. Feldt, O. Möller-Nilsson, A. I. Pavlov, Max Planck Institute for Astronomy (Germany);

J.-L. Beuzit, D. Mouillet, Lab. d'Astrophysique de l'Observatoire de Grenoble (France);

R. Gratton, Osservatorio Astronomico di Padova (Italy); M. Langlois, Lab. d'Astrophysique de Marseille (France); F. Joos, Institute of Astronomy, ETH Zürich (Switzerland)

701931 Automated HST/STIS reference file generation pipeline using OPUS [7019-121]

R. I. Diaz, M. Swam, P. Goudfrooij, Space Telescope Science Institute (United States)

701932 Bayesian approach to estimation of the map of dark current in wavelet domain [7019-123] J. Švihlík, M. Řeřábek, P. Páta, Czech Technical Univ. in Prague (Czech Republic)

701933 The lossy compression technique based on KLT [7019-124]

P. Páta, M. Řeř́ábek, Czech Technical Univ. in Prague (Czech Republic)

701934 An adaptive algorithm based on RBF for extracting the flux of fiber spectrum [7019-125] H. Qin, Z. Ye, Univ. of Science and Technology of China (China); A. Luo, H. Zhang, National Astronomical Observatories (China)

701935 Automated stellar spectral analysis software for survey spectra [7019-126] A-L. Luo, Y. Wu, J. Zhao, G. Zhao, National Astronomical Observatories (China)

701936 EVALSO: enabling virtual access to Latin American southern observatories [7019-127] R. Cirami, I. Coretti, P. Di Marcantonio, M. Pucillo, P. Santin, INAF, Osservatorio Astronomico di Trieste (Italy)

701937 An automatic system for photometric redshift estimation based on sky survey data [7019-128]

D. Wang, Y. Zhang, Y. Zhao, National Astronomical Observatories (China)

$701938 \quad$ Knowledge discovery in astronomical data [7019-129]

Y. Zhang, National Astronomical Observatories (China); H. Zheng, North China Electric Power Univ. (China); Y. Zhao, National Astronomical Observatories (China) 
701939 SPHERE data reduction and handling system: overview, project status, and development [7019-130]

A. Pavlov, O. Möller-Nilsson, M. Feldt, T. Henning, Max Planck Institute for Astronomy (Germany); J.-L Beuzit, D. Mouillet, Lab. d'Astrophysique de Grenoble (France)

7019 3A Predicting photometric redshifts with polynomial regression [7019-131]

D. Wang, Y. Zhang, Y. Zhao, National Astronomical Observatories (China)

7019 3B The oversampling mode for CoRot exo-field observations [7019-132]

C. Surace, R. Alonso, P. Barge, R. Cautain, P. Y. Chabaud, M. Delevil, T. Fenouillet,

J. C. Meunier, C. Moutou, Lab. d'Astrophysique de Marseille, CNRS, Univ. de Provence (France)

Author Index 
Downloaded From: https://www.spiedigitallibrary.org/conference-proceedings-of-spie on 26 Apr 2023

Terms of Use: https://www.spiedigitallibrary.org/terms-of-use 


\title{
Conference Committee
}

\author{
Symposium Chairs
}

Mark C. Clampin, NASA Goddard Space Flight Center (United States)

Alan F. M. Moorwood, European Southern Observatory (Germany)

Symposium Cochairs

Masanori lye, National Astronomical Observatory of Japan (Japan)

Douglas A. Simons, Gemini Observatory (United States)

Conference Chairs

Alan Bridger, UK Astronomy Technology Center (United Kingdom)

Nicole M. Radziwill, National Radio Astronomy Observatory (United States)

Program Committee

Gianluca Chiozzi, European Southern Observatory (Germany)

Kim K. Gillies, Gemini Observatory (United States) and Space Telescope Science Institute (United States)

Bret D. Goodrich, National Solar Observatory (United States)

Robert I. Kibrick, University of California Observatories, Lick Observatory (United States)

Hilton A. Lewis, California Association for Research in Astronomy (United States)

Gianni Raffi, European Southern Observatory (Germany)

David L. Terrett, Science and Technology Facilities Council (United Kingdom)

\section{Session Chairs}

1 Project Reports: Radio

Alan Bridger, UK Astronomy Technology Center (United Kingdom)

2 Telescope Control I

Gianluca Chiozzi, European Southern Observatory (Germany)

3 Telescope Control II

Hilton A. Lewis, California Association for Research in Astronomy (United States) 
$4 \quad$ Telescope Control III

Gianni Raffi, European Southern Observatory (Germany)

5 Observatory Control I

Nicole M. Radziwill, National Radio Astronomy Observatory (United States)

6 Observatory Control II

Nicole M. Radziwill, National Radio Astronomy Observatory (United States)

7 Software Frameworks

Bret D. Goodrich, National Solar Observatory (United States)

8 Project Reports: Optical/IR

Kim K. Gillies, Gemini Observatory (United States) and Space Telescope Science Institute (United States)

9 Instrument Control

Robert I. Kibrick, University of California Observatories, Lick Observatory (United States)

10 Software Engineering and Management

David L. Terrett, Science and Technology Facilities Council (United Kingdom)

11 Data Handling and Processing I

Nicole M. Radziwill, National Radio Astronomy Observatory (United States)

12 Data Handling and Processing II

Alan Bridger, UK Astronomy Technology Center (United Kingdom) 


\section{Introduction}

This year's software conference included status reports from telescopes under development, descriptions of more generalized software frameworks for easing development, and detailed descriptions of software accomplishments in the areas of instrument control, telescope control, scheduling, and observing systems. The topics ranged from design to implementation to operations, and covered design strategies, design patterns, middle-ware, data handling techniques, and approaches to quality improvement within astronomical software systems. There were many conference presentations and posters of a very high quality.

The highlight of our three days of discussion was a "town hall" style forum where we discussed the merits and challenges of sharing software, introduced by a paper on the subject (Chiozzi et al). Over 100 people attended the forum in a rather hot meeting room and there were many very good points made and issues raised. Inevitably there were differences of opinion about what can be achieved, but we were very pleased at the level of debate and thank everyone who attended. There was a strong feeling that the discussion session should be repeated in 2010.

We recognized that sharing is not just limited to the source code that drives our instruments and observing processes, but also the concepts, ideas, and dominant designs that are continually refined with each new project. Although most of us agreed that more extended collaboration should be a goal, we noted that there are financial, technical, and sociological barriers that need to be overcome. We discussed several strategies for doing this, including producing "reusable components" that attempt to solve parts of a problem rather than stimulate large-scale reuse. Between now and 2010, we aim to launch an active and sustainable discussion in this area to fuel wider, practical collaborations across the community, in addition to within the conference environment. This could include launching an online forum, developing a formal body of knowledge to support future conference publications, or other ideas that members of the community would find helpful.

We would like to thank our very active conference committee not only for their contributions to organizing the sessions, but also in their continued commitment to improving the practice of software engineering at astronomical facilities. Finally, we thank the SPIE staff for all their work and especially their patience.

\section{Alan Bridger Nicole M. Radziwill}


Downloaded From: https://www.spiedigitallibrary.org/conference-proceedings-of-spie on 26 Apr 2023

Terms of Use: https://www.spiedigitallibrary.org/terms-of-use 